\title{
STEČKIN INEQUALITIES FOR SUMMABILITY METHODS
}

\author{
JIA-DING CAO \\ Department of Mathematics \\ Fudan University \\ Shanghai, 200433, PEOPLE'S REPUBLIC OF CHINA
}

(Received April 30, 1992 and in revised form June 16, 1995)

\begin{abstract}
Stečkin proved an inequality on Fejér means of Fourier series He said, "Proving similar inequality for other summability method is an interesting problem." We generalize Steckkin's inequality and give various applications to summability methods.
\end{abstract}

KEY WORDS AND PHRASES. Stečkin inequality, M. F Timan inequality, Zygmund typical means, various summability methods.

1991 AMS SUBJECT CLASSIFICATION CODES. 41A17, 41 A45, 42A24, 42A10, 41A05, 41A40, $41 \mathrm{~A} 10$.

\section{INTRODUCTION}

Let $C_{2 \pi}$ be space of $2 \pi$-periodic continuous functions, $\|f\|:=\max _{0 \leq x \leq 2 \pi}|f(x)|$. Let $f \in C_{2 \pi}$, its Fourier series is given by

$$
f(x) \sim \frac{a_{0}}{2}+\sum_{\imath=1}^{\infty}\left(a_{\imath} \cos i x+b_{\imath} \sin i x\right) .
$$

Denote $\Pi_{n}$ to be the set of trigonometric polynomials of order at most $n$, and

$$
E_{n}(f):=E_{n}(f)_{C_{2 \pi}}:=\min _{t_{n} \in \Pi_{n}}\left\|f-t_{n}\right\| .
$$

For a triangular matrix $\wedge:=\left\{\lambda_{2, m(n)}\right\}$ with $\lambda_{0, m(n)}=1(n=0,1, \ldots)$ we consider the linear summability method

$$
\begin{aligned}
U_{m(n)}(f, x) & :=\frac{a_{0}}{2} \lambda_{0, m(n)}+\sum_{\imath=1}^{m(n)} \lambda_{\imath, m(n)}\left(a_{\imath} \cos i x+b_{\imath} \sin i x\right), \\
& =: \frac{a_{0}}{2}+\sum_{\imath=1}^{m(n)} \lambda_{\imath, m(n)} A_{\imath}(x) .
\end{aligned}
$$

If $\lambda_{2, n}=1-\frac{i}{n+1}(0 \leq i \leq n)$ we obtain Fejér means $\sigma_{n}$.

By $M_{2}$ and $C_{2}$ we denote positive constants independent of $n$, and $f$.

S. B. Stečkin proved in [1].

THEOREM A. Let $f \in C_{2 \pi}$, then we have

$$
\left\|f-\sigma_{n}(f)\right\| \leq \frac{M_{1}}{n+1} \sum_{\imath=0}^{n} E_{\imath}(f) .
$$

Let $\mathbb{N}$ be the set of natural numbers.

If $k \in \mathbb{N}$ and $\lambda_{i, n}=1-\left(\frac{i}{n+1}\right)^{k},(1 \leq i \leq n)$, we obtain Zygmund typical means $Z_{n}^{k}$

The following generalization is obtained by M. F Timan (see [2]). 
THEOREM B. Let $f \in C_{2 \pi}$, then we have

$$
\left\|f-Z_{n}^{k}(f)\right\| \leq \frac{M_{2}}{(n+1)^{k}} \sum_{\imath=0}^{n}(i+1)^{k-1} E_{\imath}(f) .
$$

In $\S 2$ we establish lemmas of comparison of summability methods with Zygmund typical means $Z_{n}^{k}$ The generalization of Stečkin's inequality is proved in $\S 3$ Using the results of $\S 2$ and $\S 3$ some applications on various summability methods are given in $\S 4-\S 5$

\section{LEMMA OF COMPARISON}

Favard and Trigub [3] investigated comparison of linear summability methods of Fourier series. Butzer, Nessel, and Trebels investigated comparison of summability methods in Banach spaces

Let $A$ be a linear operator mapping $C_{2 \pi}$ to $C_{2 \pi}$ and $\|\mathrm{A}\|$ be its norm

LEMMA 1. Suppose that $A_{n}$ is a sequence of linear operators mapping $C_{2 \pi}$ to $C_{2 \pi}$ with $\left\|A_{n}\right\|=O(1)$, and $B_{n}$ is a sequence of linear operators mapping $C_{2 \pi}$ to $\Pi_{\mathrm{n}}$ with $\left\|B_{n}\right\|=O(1)$ In order that for any $f \in C_{2 \pi}$

$$
\left\|f-A_{n} f\right\| \leq M_{3} \cdot\left\|f-B_{n} f\right\|,
$$

it is sufficient and necessary that for any $t \in \Pi_{n}$

$$
\left\|t_{n}-A_{n}\left(t_{n}\right)\right\| \leq M_{4} \bullet\left\|t_{n}-B_{n}\left(t_{n}\right)\right\| .
$$

PROOF. Necessity Obviously from (2.1) we obtain (2.2)

Sufficiency. Let $f \in C_{2 \pi}$ and $t_{n}^{*}$ be polynomial of $n$th best approximation, i.e., $\left\|f-t_{n}^{*}\right\|=E_{n}(f)$. Then by the boundness of $\left\|A_{n}\right\|$ and $\left\|B_{n}\right\|$, one gets

$$
\begin{aligned}
\left\|f-A_{n} f\right\| & \leq\left\|f-t_{n}^{*}\right\|+\left\|t_{n}^{*}-A_{n}\left(t_{n}^{*}\right)\right\|+\left\|A_{n}\left(t_{n}^{*}-f\right)\right\| \\
& \leq E_{n}(f)+M_{4} \cdot\left\|t_{n}^{*}-B_{n}\left(t_{n}^{*}\right)\right\|+M_{5} \cdot E_{n}(f) \\
& \leq\left(1+M_{5}\right) \cdot E_{n}(f)+M_{4} \cdot\left\|t_{n}^{*}-f\right\|+M_{4} \cdot\left\|f-B_{n} f\right\|+M_{4} \cdot\left\|B_{n}\left(f-t_{n}^{*}\right)\right\| \\
& \leq\left(1+M_{5}\right) \cdot E_{n}(f)+M_{4} \cdot E_{n}(f)+M_{4} \cdot\left\|f-B_{n} f\right\|+M_{4} \cdot M_{6} \cdot E_{n}(f) \\
& \leq\left(1+M_{5}+M_{4}+M_{4} \cdot M_{6}\right) \cdot E_{n}(f)+M_{4} \cdot\left\|f-B_{n} f\right\| .
\end{aligned}
$$

It is clear that if $A_{n}$ are also mapping $C_{2 \pi}$ to $\Pi_{n}$, then converse inequality holds, this is

COROLLARY 1. In Lemma 1 if in addition: $A_{n}$ is a sequence of linear operators mapping $C_{2 \pi}$ to $\Pi_{n}$, then for any $f \in C_{2 \pi}$

$$
M_{7} \cdot\left\|f-B_{n} f\right\| \leq\left\|f-A_{n} f\right\| \leq M_{8} \cdot\left\|f-B_{n} f\right\| .
$$

Corollary 1 (case in (2.2): $A_{n}\left(t_{n}\right)=B_{n}\left(t_{n}\right), \forall t_{n} \in \Pi_{n}$ ) is also obtained by Berman in [4]

LEMMA 2. Let $k \in \mathbb{N}$ and $A_{n}$ be a sequence of linear operators mapping $C_{2 \pi}$ to $C_{2 \pi}$, in order that for every $f \in C_{2 \pi}$

$$
\left\|f-A_{n} f\right\| \leq M_{9} \bullet\left\|f-Z_{n}^{k}(f)\right\|,
$$

it is sufficient and necessary that

(i) $\left\|A_{n}\right\|=O(1)$

(ii) $A_{n}$ satisfies $\left(b_{k}\right)$ (if $k$ is even) and ( $\left.\tilde{b}_{k}\right)$ (if $k$ is odd), here

Condition $\left(b_{k}\right)$ for some $k \in \mathbb{N}$ 


$$
\left\|f-A_{n} f\right\| \leq \frac{M_{10}}{(n+1)^{k}}\left\|f^{(k)}\right\|, \quad \forall f, \quad f^{(k)} \in C_{2 \pi} .
$$

Condition $\left(\tilde{b}_{k}\right)$ for some $k \in \mathbb{N}$

$$
\left\|f-A_{n} f\right\| \leq \frac{M_{11}}{(n+1)^{k}}\left\|\tilde{f}^{(k)}\right\|, \quad \forall f, \quad \tilde{f}^{(k)} \in C_{2 \pi} .
$$

Here $\tilde{f}(x)$ is a conjugate function of $f(x) \in C_{2 \pi}$

Necessity It is evident (see [5]), $\left\|Z_{n}^{k}\right\|=O(1)$, hence by (2.3) we have $\left\|A_{n}\right\|=O(1)$ The statement (ii) follows from the following Zygmund's inequalities (see [5]) in Chap. VIII, $\S 8.7$, problem 27

(iii) For $f \in C_{2 \pi}$ and $f^{(k)} \in C_{2 \pi}$

$$
\left\|f-Z_{n}^{k}(f)\right\| \leq \frac{M_{12}}{(n+1)^{k}} \cdot\left\|f^{(k)}\right\|, \quad \text { if } k \text { is even }
$$

(iv) For $f \in C_{2 \pi}$ and $\tilde{f}^{(k)} \in C_{2 \pi}$

$$
\left\|f-Z_{n}^{k}(f)\right\| \leq \frac{M_{13}}{(n+1)^{k}} \cdot\left\|\tilde{f}^{(k)}\right\|, \text { if } k \text { is odd },
$$

Sufficiency We note that for $t_{n} \in \Pi_{n}$ we have

$$
\begin{aligned}
& \frac{\left\|t_{n}^{(k)}\right\|}{(n+1)^{k}}=\left\|t_{n}-Z_{n}^{k}\left(t_{n}\right)\right\|, \quad(k \text { even }) \\
& \frac{\left\|\tilde{t}_{n}^{(k)}\right\|}{(n+1)^{k}}=\left\|t_{n}-Z_{n}^{k}\left(t_{n}\right)\right\|, \quad(k \text { odd })
\end{aligned}
$$

Combining these with (ii) we get

$$
\left\|t_{n}-A_{n}\left(t_{n}\right)\right\| \leq M_{14} \bullet\left\|t_{n}-Z_{n}^{k}\left(t_{n}\right)\right\|, \quad(k \in \mathbb{N}),
$$

the inequality (2.3) follows from this estimate and Lemma 1

\section{STEČKIN'S PROBLEM}

THEOREM 1. Suppose that $A_{n}$ is a sequence of linear operators mapping $C_{2 \pi}$ to $C_{2 \pi}, A_{n}$ satisfies $\left\|A_{n}\right\|=O(1)$ and condition $\left(\tilde{b}_{k}\right)$ for some $k \in \mathbb{N}$, then for any $f \in C_{2 \pi}$ we have

$$
\left\|f-A_{n} f\right\| \leq \frac{M_{15}}{(n+1)^{k}} \sum_{\imath=0}^{n}(i+1)^{k-1} \cdot E_{\imath}(f) .
$$

PROOF. If $k$ is odd, then (3.1) follows from Lemma 2 and Theorem B. If $k$ is even, and we choose $T_{n} \in \Pi_{n}$, such that $\left\|f-T_{n}\right\|=E_{n}(f)$, since $\left(\tilde{b}_{k}\right)$ and (2.7) $\left(Z_{n}^{1}=\sigma_{n}\right)$ we have

$$
\begin{aligned}
\left\|T_{n}-A_{n}\left(T_{n}\right)\right\| \leq \frac{M_{16}}{(n+1)^{k}}\left\|\tilde{T}_{n}^{(k)}\right\|=\frac{M_{16}}{(n+1)^{k}}\left\|\left(\tilde{T}_{n}^{(k-1)}\right)^{\prime}\right\| \\
\quad=\frac{M_{16}}{(n+1)^{k}}\left\|\left(\widetilde{T}_{n}^{(k-1)}\right)^{\prime}\right\|=\frac{M_{16}}{(n+1)^{k-1}}\left\|T_{n}^{(k-1)}-\sigma_{n}\left(T_{n}^{(k-1)}\right)\right\|,
\end{aligned}
$$


we have by Theorem $\mathrm{A}$

$$
\left\|T_{n}^{(k-1)}-\sigma_{n}\left(T_{n}^{(k-1)}\right)\right\| \leq \frac{M_{1}}{(n+1)} \sum_{\nu=0}^{n} E_{\nu}\left(T_{n}^{(k-1)}\right),
$$

to estimate the sum of (3.3), we apply the inequality (see [5]) in Chap V $\$ 5636$ If $r \in \mathbb{N}$ and $g \in C_{2 \pi}$ and $\sum_{\imath=1}^{\infty} i^{r-1} \cdot E_{\imath}(g)<\infty$, then $g^{(r)} \in C_{2 \pi}$ and

$$
E_{\nu}\left(g^{(r)}\right) \leq M_{17}\left\{(\nu+1)^{r} \cdot E_{\nu}(g)+\sum_{\imath=\nu+1}^{\infty} i^{r-1} \cdot E_{\imath}(g)\right\},
$$

taking $r=k-1(k \geq 2), g=T_{n}$, since $E_{\imath}\left(T_{n}\right)=0(i \geq n)$ we obtain for $0 \leq \nu \leq n$

$$
E_{\nu}\left(T_{n}^{(k-1)}\right) \leq M_{17}\left\{(\nu+1)^{k-1} \cdot E_{\nu}\left(T_{n}\right)+\sum_{i=\nu}^{n} i^{k-2} \cdot E_{\imath}\left(T_{n}\right)\right\}
$$

for $0 \leq i \leq n$ from definition we have

$$
E_{2}(g) \leq\|g-0\|=\|g\|, \quad \forall g \in C_{2 \pi},
$$

and we have (see [5]) in Chap II, §2.5

(1) for $g$ and $h \in C_{2 \pi}, E_{\imath}(g+h) \leq E_{\imath}(g)+E_{\imath}(h)$;

(2) $E_{n}(f) \leq E_{\imath}(f)$

hence

$$
E_{\imath}\left(T_{n}\right) \leq E_{\imath}\left(T_{n}-f\right)+E_{i}(f) \leq\left\|T_{n}-f\right\|+E_{\imath}(f)=E_{n}(f)+E_{\imath}(f) \leq 2 E_{\imath}(f),
$$

from (3.5) we have

hence

$$
E_{\nu}\left(T_{n}^{(k-1)}\right) \leq 2 M_{17} \cdot\left\{(\nu+1)^{k-1} \cdot E_{\nu}(f)+\sum_{i=\nu}^{n} i^{k-2} \cdot E_{\imath}(f)\right\},
$$

we have

$$
\sum_{\nu=0}^{n} E_{\nu}\left(T_{n}^{(k-1)}\right) \leq 2 M_{17} \cdot\left\{\sum_{\nu=0}^{n}(\nu+1)^{k-1} \cdot E_{\nu}(f)+\sum_{\nu=0}^{n} \sum_{\imath=\nu}^{n} i^{k-2} \cdot E_{\imath}(f)\right\},
$$

$$
\sum_{\nu=0}^{n} \sum_{\imath=\nu}^{n} i^{k-2} \cdot E_{\imath}(f)=\sum_{\imath=0}^{n} \sum_{\nu=0}^{\imath} i^{k-2} \cdot E_{\imath}(f)=\sum_{\imath=0}^{n} i^{k-2} \cdot(i+1) \cdot E_{\imath}(f) \leq \sum_{\imath=0}^{n}(i+1)^{k-1} \cdot E_{\imath}(f),
$$

combining (3.2), (3.3), (3.6), (3.7) we have

$$
\left\|T_{n}-A_{n}\left(T_{n}\right)\right\| \leq \frac{4 M_{16} \cdot M_{1} \cdot M_{17}}{(n+1)^{k}} \sum_{i=0}^{n}(i+1)^{k-1} \cdot E_{\imath}(f),
$$

since $\left\|A_{n}\right\|=O(1)\left(\left\|A_{n}\right\| \leq M_{18}\right)$ we have

$$
\begin{aligned}
\left\|f-A_{n} f\right\| & \leq\left\|f-T_{n}\right\|+\left\|T_{n}-A_{n}\left(T_{n}\right)\right\|+\left\|A_{n}\left(T_{n}-f\right)\right\| \\
& \leq E_{n}(f)+\frac{4 M_{16} \cdot M_{1} \cdot M_{17}}{(n+1)^{k}} \sum_{\imath=0}^{n}(i+1)^{k-1} \cdot E_{\imath}(f)+M_{18} \cdot E_{n}(f) .
\end{aligned}
$$




$$
\begin{aligned}
E_{n}(f) & \leq \frac{c_{1}}{(n+1)^{k}} \sum_{i=0}^{n}(i+1)^{k-1} \cdot E_{n}(f) \\
& \leq \frac{c_{1}}{(n+1)^{k}} \sum_{\imath=0}^{n}(i+1)^{k-1} \cdot E_{\imath}(f),
\end{aligned}
$$

combining (39) and (3.10) we get

$$
\left\|f-A_{n} f\right\| \leq \frac{M_{15}}{(n+1)^{k}} \sum_{\imath=0}^{n}(i+1)^{k-1} \cdot E_{\imath}(f) .
$$

REMARK 1. Let $k \in \mathbb{N}$ and $A_{n}$ be a sequence of linear operators mapping $C_{2 \pi}$ to $C_{2 \pi}$ In order that for any $f \in C_{2 \pi}$

$$
\left\|f-A_{n} f\right\| \leq M_{19} \cdot \omega_{k}\left(f, \frac{1}{n}\right)
$$

it is sufficient and necessary that $A_{n}$ satisfies conditions $\left\|A_{n}\right\|=O(1)$ and $\left(b_{k}\right)$ (see [6]) on page 182

We have (see [5]) in Chap. VI, 6.11, for $f \in C_{2 \pi}$

$$
\omega_{k}\left(f, \frac{1}{n}\right) \leq \frac{M_{20}}{n^{k}} \sum_{\imath=0}^{n}(i+1)^{k-1} \cdot E_{\imath}(f),
$$

from Remark 1 we also obtain

$$
\left\|f-A_{n} f\right\| \leq \frac{M_{21}}{(n+1)^{k}} \sum_{\imath=0}^{n}(i+1)^{k-1} \cdot E_{\imath}(f) .
$$

Let $f \in C_{2 \pi}$ and $\omega_{1}(f, \delta)$ be the modulus of continuity of $f$. Classes of functions $\operatorname{Lip}(1, M)$ : $=\left\{f \mid \omega_{1}(f, \delta) \leq M \delta\right\}$, and Lip 1: $=\left\{\bigcup_{M>0} \operatorname{Lip}(1, M)\right\}$

LEMMA 3. Let $A_{n}$ be a sequence of linear operators mapping $C_{2 \pi}$ to $C_{2 \pi}, A_{n}(1, x)=1$, if for $\tilde{f}^{(k-1)} \in \operatorname{Lip} 1$

$$
\left\|f-A_{n} f\right\|=O\left(\frac{1}{n^{k}}\right)
$$

then for $f \in C_{2 \pi}$ and $\tilde{f}^{(k)} \in C_{2 \pi}$ we have

$$
\left\|f-A_{n} f\right\| \leq \frac{M_{22}}{(n+1)^{k}} \cdot\left\|\tilde{f}^{(k)}\right\| .
$$

PROOF. If $f \in C_{2 \pi}$ and $\tilde{f}^{(k)} \in C_{2 \pi}$ we have

$$
\left|\tilde{f}^{(k-1)}(x+t)-\tilde{f}^{(k-1)}(x)\right| \leq\left\|\tilde{f}^{(k)}\right\| t, \quad(t \geq 0),
$$

if $D:=\left\|\tilde{f}^{(k)}\right\|>0$ then $\tilde{f}^{(k-1)} / D \in \operatorname{Lip}(1,1)$, from (3.11) we obtain $\left\|\frac{f}{D}-A_{n}\left(\frac{f}{D}\right)\right\|=O\left(\frac{1}{n^{k}}\right)$, hence

$$
\left\|f-A_{n} f\right\| \leq \frac{M_{22}}{(n+1)^{k}} \cdot D=\frac{M_{22}}{(n+1)^{k}}\left\|\tilde{f}^{(k)}\right\|
$$

if $\left\|\tilde{f}^{(k)}\right\|=0$, then $f \equiv$ const (see [5]) in $§ 5.91$, since $A_{n}(1, x)=1$, obviously (3.12) holds 
Sequence of Fejér mean $\sigma_{n}$ is saturated with order $\left(n^{-1}\right)$ and saturation class $S\left(L_{n}\right):=\{f \mid \tilde{f} \in$ Lip $1\}$, using Lema 3 we obtain that $\sigma_{n}$ satisfies $\left(\tilde{b}_{1}\right)$, since $\left\|\sigma_{n}\right\|=1$ and Theorem 1, we obtain Theorem A

PROBLEM 1. Let $A_{n}$ be a sequence of linear operators mapping $C_{2 \pi}$ to $C_{2 \pi}$, finding sufficient and necessary conditions on $A_{n}$ such that Timan type inequality (1 3 ) holds

\section{APPLICATIONS}

We give applications on linear summability method $\bigcup_{m(n)}$ of Fourier series Firstly we have $\bigcup_{m(n)}(1, x)=1$

EXAMPLE 1. $(C, \alpha)$ means $\sigma_{n}^{\alpha}(\alpha>0) . \quad \lambda_{\imath, n}=\frac{A_{n-1}^{\alpha}}{A_{n}^{\alpha}}(0 \leq i \leq n), A_{n}^{\alpha}:=\frac{(\alpha+1)(\alpha+2)(\alpha+n)}{n^{\prime}}$

Trigub proved [3]

LEMMA 4. Let $\alpha>0$ and $f \in C_{2 \pi}$, then

$$
C_{3} \bullet\left\|f-\sigma_{n}(f)\right\| \leq\left\|f-\sigma_{n}^{\alpha}(f)\right\| \leq C_{2} \bullet\left\|f-\sigma_{n}(f)\right\| .
$$

THEOREM 2. Let $\alpha>0$ and $f \in C_{2 \pi}$, then we have

$$
\left\|f-\sigma_{n}^{\alpha}(f)\right\| \leq \frac{M_{23}}{n+1} \sum_{\imath=0}^{n} E_{\imath}(f) .
$$

PROOF. Obviously from Theorem A and Lemma 4 we obtain Theorem 2.

Let $\omega(\delta)$ be a modulus of continuity and $\omega(\delta)>0(0<\delta \leq \pi)$. Class of functions $H_{\omega}:=\left\{f \mid \omega_{1}(f, \delta) \leq \omega(\delta), 0 \leq \delta \leq \pi\right\}$.

Let $\omega_{1}^{* *}(\delta)$ be a modified function of first order of $\omega(\delta)$ (see [1])

$$
\omega_{1}^{* *}(\delta):=\delta \cdot \inf _{0<\eta \leq \delta}\left\{\eta^{-1} \cdot \inf _{\eta \leq \xi \leq \pi} \omega(\xi)\right\} \text {, we have } \omega_{1}^{* *}(\delta) \leq \omega(\delta) .
$$

Let $a_{n}>0, b_{n}>0, a_{n} \approx b_{n}$ means that there are $C_{4}>0, C_{5}>0$ such that $C_{4} a_{n} \leq b_{n} \leq C_{5} a_{n}$.

COROLLARY 2. Let $\alpha>0$, we have

$$
\begin{aligned}
& \sup _{f \in H_{\omega}}\left\|f-\sigma_{n}^{\alpha}(f)\right\| \approx \frac{1}{n} \sum_{i=1}^{n} \omega^{* *}\left(\frac{1}{i}\right), \\
& \sup _{f \in H_{\omega}}\left\|f-\sigma_{n}^{\alpha}(f)\right\| \approx \frac{1}{n} \sum_{i=1}^{n} \omega\left(\frac{1}{i}\right), \\
& \sup _{f \in H_{\omega}}\left\|f-\sigma_{n}^{\alpha}(f)\right\| \approx \frac{1}{n} \int_{\frac{1}{n}}^{\pi} \frac{\omega(u)}{u^{2}} d u .
\end{aligned}
$$

PROOF. For (4.2) $(\alpha \geq 1)$ see Sun [7]. For (4.3) $(\alpha=1)$ see Devore [8] on page 227 For (4.4) $(\alpha \geq 1)$ see Mazhar and Totik [9]. Using Lemma 4 we have Corollary 2.

Stečkin also proved (see [1])

LEMMA 5. For $f$ and $\tilde{f} \in C_{2 \pi}$, we have

$$
\left\|f-\sigma_{n}(f)\right\|=O\left(E_{n}(f)\right)+O\left(\omega_{1}\left(\tilde{f}, \frac{1}{n}\right)\right) .
$$

Lemma 4 implies 
COROLLARY 3. Let $\alpha>0$, for $f$ and $\tilde{f} \in C_{2 \pi}$ we have

$$
\left\|f-\sigma_{n}^{\alpha}(f)\right\|=O\left(E_{n}(f)+O\left(\omega_{1}\left(\tilde{f}, \frac{1}{n}\right)\right) .\right.
$$

(4 2) and (4 5) answer two problems of Sun [7] on $\sigma_{n}^{\alpha}(0<\alpha<1)$

EXAMPLE 2. M Riesz means $R_{n}^{(\lambda, \delta)} \quad \lambda_{\imath, n}=\lambda\left(\frac{i}{n+1}\right)(0 \leq i \leq n), \lambda(u)=\left(1-u^{\lambda}\right)^{\delta}(\lambda \in \mathbb{N}, \delta>0)$

B Nagy proved that (see [5]) in Chap VIII, $\S 87$, problem 13, $\left\|R_{n}^{(\lambda, \delta)}\right\|=O(1)$ G Sunouchi proved that [6] on page $72, R_{n}^{(\lambda, \delta)}$ is saturated with order $\left(n^{-\lambda}\right)$ and the saturation class is $S\left(R_{n}^{(\lambda, \delta)}\right):=\left\{f \mid \tilde{f}^{(\lambda-1)} \in \operatorname{Lip} 1(\lambda\right.$ odd $)$ and $f^{(\lambda-1)} \in \operatorname{Lip} 1(\lambda$ even $\left.)\right\}$, using Theorem 1 and Remark 1 we obtain that for any $f \in C_{2 \pi}$

$$
\left\|f-R_{n}^{(\lambda, \delta)}(f)\right\| \leq \frac{M_{24}}{(n+1)^{\lambda}} \sum_{\imath=0}^{n}(i+1)^{\lambda-1} \cdot E_{\imath}(f), \quad(\lambda \in \mathbb{N}) .
$$

EXAMPLE 3. Operators $L_{n}$ determined by convolution with kernels of Korovkin (see [8]) on page $107 L_{n}$ is saturated with order $\left(n^{-1}\right)$ and saturation class $S\left(L_{n}\right):=\{f \mid \tilde{f} \in \operatorname{Lip} 1\}$, hence we obtain Stečkin type inequality

EXAMPLE 4. Nishishiraho and Wang Si-Lei proved (see [10])

LEMMA 6. Suppose that there exists a sequence $\left\{\phi_{n}\right\}$ of positive real numbers converging to zero, which satisfies

$$
\lim _{n \rightarrow \infty} \frac{\left(1-\lambda_{\imath, n}\right)}{\phi_{n}}=K, \quad \text { and let } \sum_{i=0}^{n}\left|\triangle^{2} \lambda_{\imath, n}\right|=0\left(\phi_{n}\right) \text {, }
$$

where $\triangle^{2} \lambda_{2, n}=\lambda_{2, n}-2 \lambda_{2+1, n}+\lambda_{2+2, n}$, and $\lambda_{2, n}=0(i>n)$. If $\phi_{n}=\frac{6}{n}$, then $\bigcup_{n}$ is saturated with the order $\left(n^{-1}\right)$ and saturation class $S\left(\bigcup_{n}\right):=\{f \mid \tilde{f} \in \operatorname{Lip} 1\}$, using Theorem 1 and Lemma 3 we obtain Stečkin type inequality.

\section{POLYNOMIALS OF INTERPOLATION AND CAO-GONSKA OPERATORS}

Let $f(x) \in C_{2 \pi}$ and $\bigcup_{n}^{*}(f, x)$ be linear summability (with $\wedge=\left\{\lambda_{2, n}\right\}$ ) of trigonometric polynomial of interpolation on nodes $y_{2}=\frac{22 \pi}{2 n+1}(i=0,1, \ldots, 2 n)$ [4] [5]. Berman proved [4] and [5] in 8.7, problem 7

LEMMA 7. Let $K_{n}(v):=\frac{1}{2}+\sum_{\imath=1}^{n} \lambda_{\imath, n} \cos i v, \int_{0}^{\pi}\left|K_{n}(v)\right| d v=O(1)$, then for $f \in C_{2 \pi}$

$$
M_{25} \cdot\left\|f-U_{n}(f)\right\| \leq\left\|f-U_{n}^{*}(f)\right\| \leq M_{26} \cdot\left\|f-U_{n}(f)\right\| .
$$

THEOREM 3. Let $k \in \mathbb{N}$ and $\int_{0}^{\pi}\left|K_{n}(v)\right| d v=O(1)$, and $\wedge=\left\{\lambda_{i, n}\right\}$ satisfies $\left(\tilde{b}_{k}\right)$, then, for any $f \in C_{2 \pi}$

$$
\left\|f-U_{n}^{*}(f)\right\| \leq \frac{M_{27}}{(n+1)^{k}} \sum_{\imath=0}^{n}(i+1)^{k-1} E_{\imath}(f) .
$$

PROOF. From Lemma 7 and Theorem 1 we obtain Theorem 3.

Let $f \in C[-1,1]$, the Pičugov-Lehnhoff operators are defined by $(\theta=\arccos x, x \in[-1,1]$, $\left.K_{m(n)}(v):=\frac{1}{2}+\sum_{i=1}^{m(n)} \lambda_{2, m(n)} \cos i v\right)$

$$
G_{m(n)}(f(t), x):=\frac{1}{\pi} \int_{-\pi}^{\pi} f(\cos (v+\arccos x)) K_{m(n)}(v) d v .
$$


Let $T_{\imath}(x):=\cos (\imath \arccos x)$ be the $\mathrm{i}$-th Cebys̃ev polynomial, and $x_{\gamma, N_{0}}:=\cos \frac{2 \gamma-1}{2 N_{0}} \pi$, $1 \leq \gamma \leq N_{0}$, the Cao and Gonska polynomials are defined by (see [11])

$$
\wedge_{m(n), N_{0}}(f, x):=\frac{1}{N_{0}} \sum_{\gamma=1}^{N_{0}} f\left(x_{\gamma, N_{0}}\right)\left\{1+2 \sum_{i=1}^{m(n)} \lambda_{\imath, m(n)} \bullet T_{\imath}\left(x_{\gamma, N_{0}}\right) \cdot T_{\imath}(x)\right\}
$$

specifically $\wedge_{n-1, n}$ are the Varma-Mills operators (see [11])

LEMMA 8. Let $N_{0} \geq m(n)+1$ and $\int_{0}^{\pi}\left|K_{m(n)}(v)\right| d v=O(1)$, then for any $f \in C[-1,1]$

$$
M_{28} \bullet\left\|f-G_{m(n)}(f)\right\|_{C[-1,1]} \leq\left\|f-\wedge_{m(n), N_{0}}(f)\right\|_{C[-1,1]} \leq M_{29} \bullet\left\|f-G_{m(n)}(f)\right\|_{C[-1,1]} .
$$

PROOF. (see [12]).

THEOREM 4. Let $k \in \mathbb{N}, N_{0} \geq m(n)+1$, and $\wedge=\left\{\lambda_{\imath, m(n)}\right\}$ satisfies $\int_{0}^{\pi}\left|K_{m(n)}(v)\right| d v=O(1)$ and $\left(\tilde{b}_{k}\right)$, then for any $f \in C[-1,1]$

$$
\left\|f-\wedge_{m(n), N_{0}}(f)\right\|_{C[-1,1]} \leq \frac{M_{30}}{(n+1)_{K}} \sum_{i=0}^{n}(i+1)^{k-1} \cdot E_{\imath}(f)_{C[-1,1]} .
$$

PROOF. Letting $\phi(t)=f(\cos t)$, using Lemma 8 and Theorem 1 we obtain Theorem 4 .

ACKNOWLEDGMENT. The author expresses his thanks to Professor Heinz $\mathrm{H}$ Gonska from European Business School, Germany for his support The author would like to thank the referee for his/her helpful suggestions and comments.

\section{REFERENCES}

[1] STEČKIN, S B., The approximation of periodic functions by Fejér sums (Russian), Trudy Matem Instituta im V. A. Steklova 62 (1961), 48-60; Amer. Math. Soc. Translations (2) 28 (1963), 269282.

[2] TIMAN, M F., Best approximation of functions and linear methods of summability of Fourier series (Russian), Izv. Akad. Nauk. SSSR, Ser. Matem. 29 (1965), 587-604

[3] TRIGUB, R. M., Linear methods of summability and absolute convergence of Fourier series (Russian), Izv. Akad. Nauk. SSSR. Ser. Matem. 32 (1968), 24-49.

[4] BERMAN, D. L., Some remarks on the problem of the degree of approximation of polynomial operators (Russian), Izv. Vyssh. Uchebn, Zaved. Mat. 5 (1961), 3-5.

[5] TIMAN, A. F., Theory of Approximation of Functions of a Real Variable, Macmillan, New York, 1963.

[6] BUTZER, P. L. and KOREVAAR, J., On Approximation Theory, Proceedings of the Conference 1963, Birkhäuser Verlag, 1964.

[7] SUN, JUN-SEN, Uniform approximation of continuous periodic functions by Cesàro means of their Fourier series (Chinese), Advances in Math. 6 (1963), 379-387.

[8] DEVORE, R. A., The Approximation of Continuous Functions by Positive Linear Operators, Berlin-Heidelberg-New York. Springer, 1972.

[9] MAZHAR, S. M and TOTIK, V., Approximation of continuous functions by T-means of Fourier series, J. Approx. Theory 60 (1990), 174-182.

[10] WANG, SI-LEI, Saturation of trigonometric polynomial operators (Chinese), J. of Hangzhou Univ. (Nat Edition) 8 (1981), 7-13.

[11] CAO, JIA-DING and GONSKA, H H., Approximation by Boolean sums of positive linear operators III: Estimates for some numerical approximation schemes, Numer. Funct. Anal. and Optimiz. 10 (7 \& 8) (1989), 643-672.

[12] CAO, JIA-DING and GONSKA, H. H., Solutions of Butzer's problem (linear form) and some linear algebraic polynomial operators with saturation order $O\left(n^{-2}\right)$, submitted for publication 


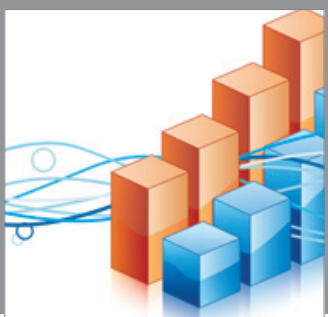

Advances in

Operations Research

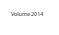

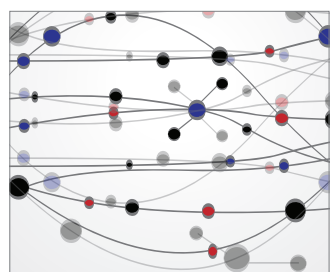

\section{The Scientific} World Journal
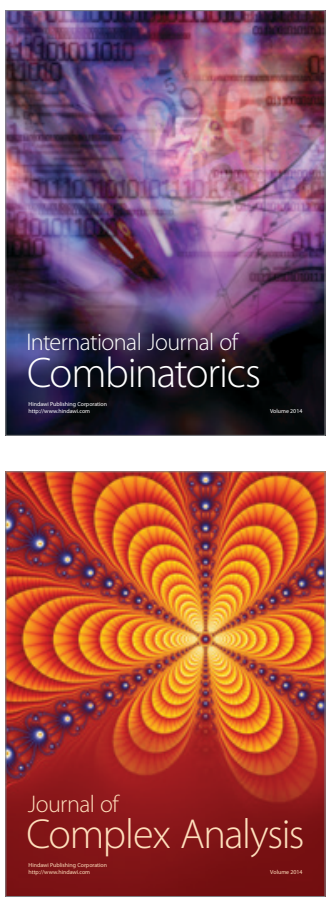

International Journal of

Mathematics and

Mathematical

Sciences
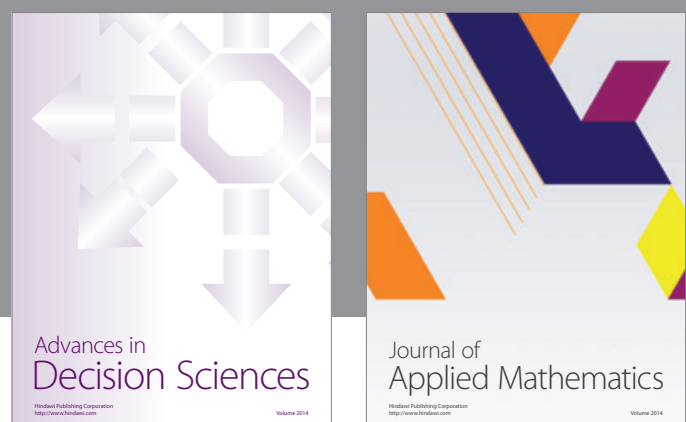

Journal of

Applied Mathematics
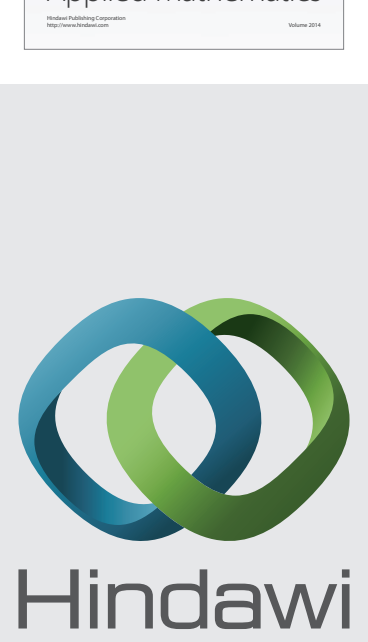

Submit your manuscripts at http://www.hindawi.com
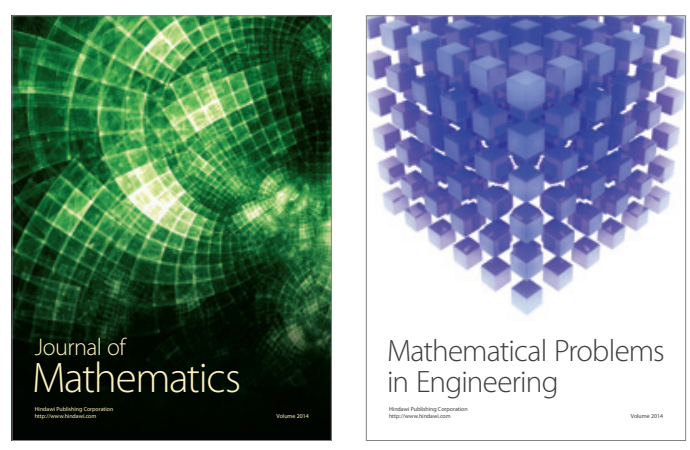

Mathematical Problems in Engineering
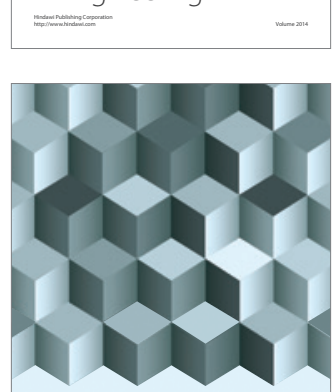

Journal of

Function Spaces
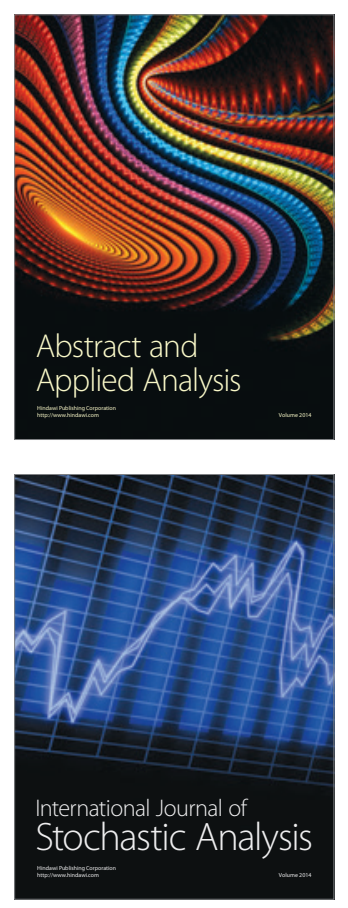

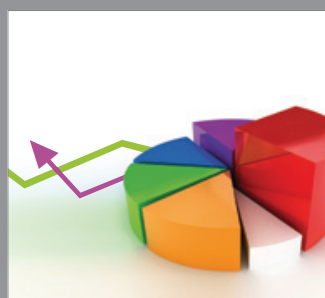

ournal of

Probability and Statistics

Promensencen
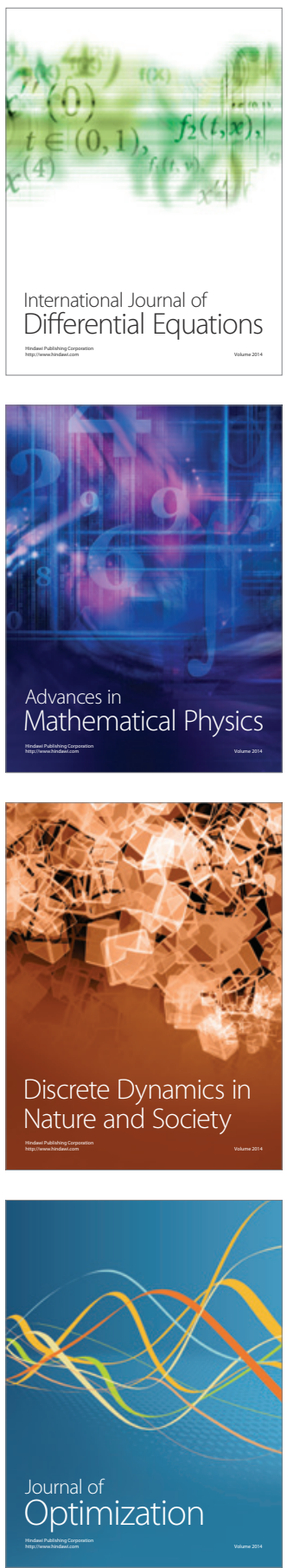